\title{
Stochastic Sequential Modeling: Toward Improved Prostate Cancer Diagnosis Through Temporal-Ultrasound
}

\author{
Layan Nahlawi (i), ${ }^{1}$ Farhad Imani, ${ }^{2}$ Mena Gaed, ${ }^{3}$ Jose A. Gomez, ${ }^{3}$ \\ Madeleine Moussa, ${ }^{3}$ Eli Gibson, ${ }^{4}$ Aaron Fenster, ${ }^{5}$ Aaron Ward, ${ }^{6}$ \\ Purang Abolmaesumi, ${ }^{2}$ Parvin Mousavi, ${ }^{1}$ and Hagit Shatkay ${ }^{1,7}$
}

\begin{abstract}
${ }^{1}$ School of Computing, Queen's University, Kingston, ON K7L 2N8, Canada; ${ }^{2}$ Department of Electrical and Computer Engineering, University of British Columbia, Vancouver, BC, Canada; ${ }^{3}$ London Health Sciences Centre, London, ON, Canada; ${ }^{4}$ University College London, London, UK; ${ }^{5}$ Robarts Research Institute, London, ON, Canada; ${ }^{6}$ Department of Medical Physics, Western University, London, ON, Canada; and ${ }^{7}$ Department of Computer and Information Sciences, University of Delaware, Newark, DE, USA
\end{abstract}

(Received 11 November 2019; accepted 27 July 2020; published online 10 August 2020)

Associate Editor Agata A. Exner oversaw the review of this article.

\begin{abstract}
Prostate cancer (PCa) is a common, serious form of cancer in men that is still prevalent despite ongoing developments in diagnostic oncology. Current detection methods lead to high rates of inaccurate diagnosis. We present a method to directly model and exploit temporal aspects of temporal enhanced ultrasound (TeUS) for tissue characterization, which improves malignancy prediction. We employ a probabilistic-temporal framework, namely, hidden Markov models (HMMs), for modeling TeUS data obtained from PCa patients. We distinguish malignant from benign tissue by comparing the respective log-likelihood estimates generated by the HMMs. We analyze 1100 TeUS signals acquired from 12 patients. Our results show improved malignancy identification compared to previous results, demonstrating over $85 \%$ accuracy and AUC of 0.95 . Incorporating temporal information directly into the models leads to improved tissue differentiation in PCa. We expect our method to generalize and be applied to other types of cancer in which temporal-ultrasound can be recorded.
\end{abstract}

Keywords-Image guided diagnosis, Hidden Markov models, Time-domain analysis, TRUS-guided biopsies, Tissue characterization.

Address correspondence to Layan Nahlawi, School of Computing, Queen's University, Kingston, ON K7L 2N8, Canada. Electronic mail: layan.nahlawi@queensu.ca

Parvin Mousavi and Hagit Shatkay have contributed equally to this work and sharing last authorship.

\section{INTRODUCTION}

Prostate cancer $(\mathrm{PCa})$ is the most commonly diagnosed form of cancer in men, second only to skin cancer. The number of new cases in the USA alone during 2019 is estimated at $174,650{ }^{1}$ A definitive diagnosis is obtained through histopathology analysis of prostate-tissue specimen collected during core needle biopsy under trans-rectal ultrasound (TRUS) guidance after initial clinical-assessment. ${ }^{7}$ Some centers use magnetic resonance (MR) and MR-TRUS fusion for guiding biopsies. ${ }^{22,28}$ TRUS-guided biopsies often lead to a high rate $(\sim 40 \%)$ of false negatives for cancer diagnosis. ${ }^{27}$ Extensive heterogeneity in morphology and pathology of $\mathrm{PCa}$ are challenging factors for accurate diagnosis and grading of the disease. ${ }^{4}$ While improved PCa screening has reduced mortality rates by $45 \%$ over the past two decades, ${ }^{5,8}$ inaccurate diagnosis and grading lead to an increase in repeat biopsies, over-diagnosis and over-treatment. ${ }^{10,16}$ Overaggressive treatment of $\mathrm{PCa}$ patients results in a decline in their quality of life.

For indolent $\mathrm{PCa}$, such aggressive treatment should be avoided, as watchful waiting and active surveillance have proven effective as disease management options. ${ }^{26}$ Accurate identification and grading of lesions and their extent - especially using affordable, readily accessible technology such as ultrasound - can, therefore, significantly contribute to appropriate effective treatment. To achieve this, methods must be developed to guide 
clinicians during biopsies to target regions with high risk of being malignant. The task of differentiating malignant tissue from its surrounding tissue is referred to in the literature as tissue typing or characterization.

Different imaging modalities have been employed for tissue characterization including ultrasound-based techniques, ${ }^{2,12,14,17-20}$ magnetic resonance sequences. ${ }^{22,28}$ Despite the low resolution of ultrasound images, ultrasound-based techniques have the advantage of using a low-cost technology that is already integrated into standard diagnostic procedures. Temporal enhanced ultrasound (TeUS) is a novel ultrasound-based imaging technique, where a sequence of ultrasound frames is captured by sonicating tissue over a short period of time, without intentionally moving the tissue or the ultrasound probe. We propose to improve the differentiation between malignant and benign prostatic tissues by capturing the temporality of the data using Hidden Markov models (HMMs). Our approach characterizes the distinct temporal signatures of echo-response from malignant and benign tissues and uses the identified signatures to detect malignancy.

Previous research on TeUS utilized frequency-domain analysis and classifiers such as support vector machines, deep belief networks and others. ${ }^{2,12,17}$ In our recent studies, we have proposed to directly represent the temporality of TeUS and employ it to reach more accurate tissue-typing, ${ }^{18-20}$ and showed preliminary results when applied to a small dataset.

Here we present in detail our stochastic approach for directly representing the temporal aspects of TeUS, through $\mathrm{HMMs},{ }^{24}$ while applying it to a larger number of patients and increased amount of available data. Importantly, this approach allows us to assess the impact of model parameters on the clinical translation of TeUS. Probabilistic temporal modeling, particularly HMMs, have been applied to a wide range of clinical data such as time dependent physiological process, ${ }^{6,30}$ and disease-risk progression over time. ${ }^{11,15} \mathrm{We}$ apply our method to differentiate between malignant vs. benign prostate tissue and demonstrate its utility, showing improved performance compared to the stateof-the-art.

Employing parsimonious models that have a relatively small number of parameters for TeUS-based characterization facilitates efficient real-time implementation that can be integrated into current clinical procedures while avoiding major interruption to the diagnostic workflow. We investigate the impact of several design decisions involved in modeling via HMMs on tissue typing performance and discuss how the choice of model parameters affects the classification outcome. We also provide a statistical comparison of our outcome with previous results reported by Imani et al., ${ }^{12}$ who used spectral features and applied support vector machines on the same TeUS signals, without explicitly modeling the temporality of the data as we do here. This comparison further demonstrates the value of such temporal modeling.

The rest of the paper is organized as follows: Sect. "Materials and Methods" describes TeUS data and its representation; it also presents the tissue-characterization framework; Sect. "Results" explains our experiments and results demonstrating the effectiveness of the method, and Sect. "Discussion" provides a discussion and concludes by outlining future work.

\section{MATERIALS AND METHODS}

\section{Data Collection}

TeUS signals record tissue-response to prolonged sonication in comparison with conventional US imaging standards. These responses consist of reflected ultrasound echointensity values. Echointensities vary over time due to changes in the microstructure of scatterers (cell nuclei) induced by external or internal vibrations such as pulsation. ${ }^{3}$ TeUS signals have been shown to carry tissue-specific information relayed by the patterns of change in echointensity over time. ${ }^{19}$ Fig. 1 shows ultrasound image-frames collected from prostate sonication (each frame is referred to as a radio frequency (RF) frame). The boundary of the prostate is encircled in a solid line (white); the red solid dots indicate the same location within the prostate over time, while the blue dotted arrows point to the corresponding echo intensity values. The sequence of echo intensities obtained from the same point within the prostate over time forms the TeUS signal (bottom of Fig. 1). We partition each RF-frame into a grid of smaller regions, each as wide as the specimen-collection needle $(16$ gauge $=1.65 \mathrm{~mm}) \cdot{ }^{13,21}$ Each window in the grid is referred to as a region of interest (ROI) and comprises multiple RF values. We use the same dataset as Imani et al. ${ }^{12}$ to ensure a valid comparison between our results and those described in their previous work. The RF-frame size is $55 \times 50 \mathrm{~mm}$, corresponding to $1276 \mathrm{RF}$ values in the axial direction and $64 \mathrm{RF}$ values in the lateral direction. Accordingly, we adopt the same ROI size $1.7 \times 1.7 \mathrm{~mm},{ }^{2}$ which corresponds to $44 \mathrm{RF}$ samples in the axial direction and $2 \mathrm{RF}$ lines in the lateral direction.

The image data consists of in vivo RF-frames gathered from $12 \mathrm{PCa}$ patients who have undergone radical prostatectomy. The study was approved by the institutional research ethics board and informed consent was obtained from all participants. Prior to the surgery, $128 \mathrm{RF}$-frames recorded at a rate of 77 frames/sec along the parasagittal plane, were gathered 
(a) Lateral

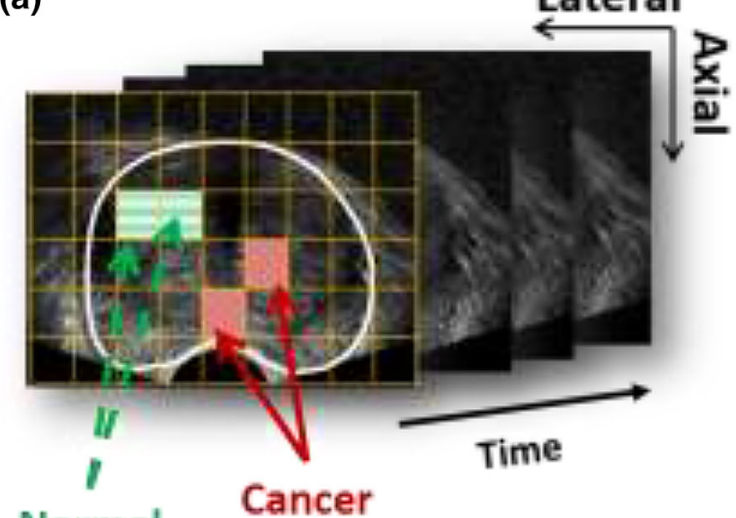

Normal

ROls

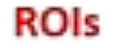

(b)

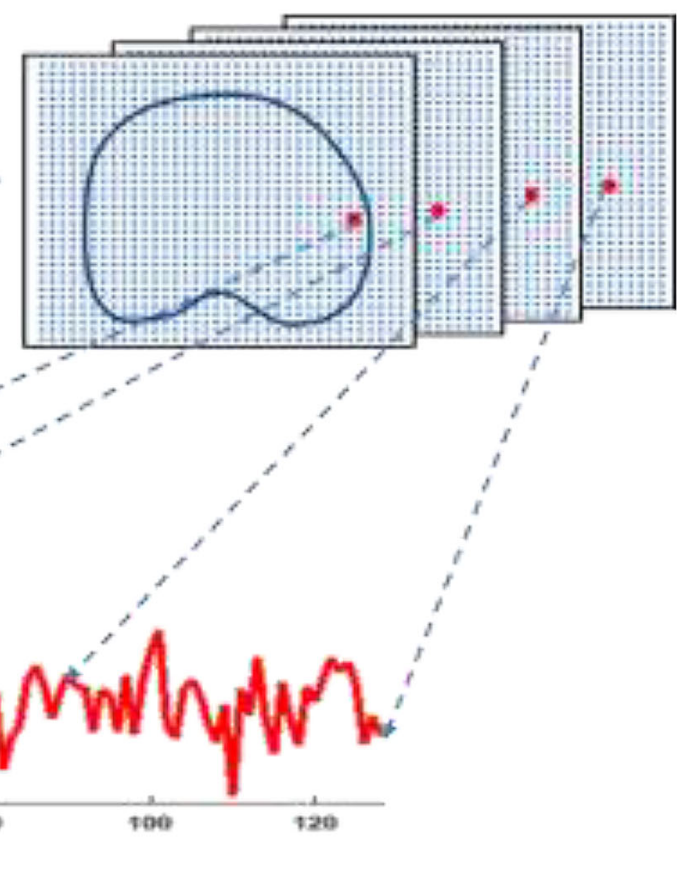

FIGURE 1. (a) Ultrasound RF-frames collected from a prostate-cancer patient over time. A grid divides each frame into ROIs. The solid red arrows point to ROls labeled as malignant, while the dashed green arrows point to ROls labeled as benign. (b) The corresponding echointensity values; the solid red dots indicate the same location across multiple frames. (c) The time series, TeUS signal for this location is shown at the bottom.

from each patient using a side-firing transrectal probe (BPL9-5/55 transrectal probe, Analogic, MA, USA) with $6.67 \mathrm{MHz}$ central frequency. The data was acquired as a fan of $2 \mathrm{D}$ ultrasound $\mathrm{RF}$ time series using a $2^{\circ}$ rotational interval between consecutive spatial slices, for approximately $2 \mathrm{~s}$ per angle. A grid was overlaid on each of the frames and ROIs were obtained as described above. To create the ground truth for malignant vs. benign regions, we used wholemount histopathology information.

Following prostatectomy, the tissues were imaged using MRI, then analyzed through high resolution microscopy; two clinicians assigned (in consensus) the appropriate labels to the ROIs within each slice. ${ }^{9,12}$ The registration of ultrasound images and high-resolution histopathology images is a challenging procedure, where MR was used as an intermediary modality. ${ }^{9,12}$ A multi-step registration process, in which MRI images are used as an intermediate step, ${ }^{9,31}$ was employed to overlay the labeled histopathology images on the in vivo ultrasound frames (see Ref. 12 for additional details). Figure 2 serves as visualization of the various registration steps needed to overlap the histopathology demarcations on the in vivo ultrasound slices. Figure 2a is a depiction of the intersection of between the ultrasound imaging planes (parasagital planes) and the histopathology cross-sections. Figure $2 \mathrm{~b}$ shows the ultrasound label-map where the prostate is segmented and the intersection lines of histopathology cross-sections are shown as oblique lines along with a registered malignant demarcation shown as white colored pixels and red-encircled. Figure $2 \mathrm{c}$ is an example of an ex vivo MRI image with visible fiducials serving as points of reference to register histopathology demarcations on ex vivo MR. Figure $2 \mathrm{~d}$ is an example of histopathology cross-section, where black is used to demarcate the malignant region and encircled in red. This malignant demarcation corresponds to the red-encircled region on the US label-map. This registration overlays true pathology labels on each ROI, indicating whether it is malignant or benign. ROIs with other non-malignant annotations such as Benign Prostatic Hyperplasia (BPH) were not included in the analysis. The ROI selection was guided by the availability of ground truth label. Figure 1 shows several examples of labeled ROIs. Benign ROIs were chosen from areas with no histopathology demarcation, and with a safe margin of $\geq 5 \mathrm{~mm}$ away from malignancy and other conditions. The malignant ROIs were picked from demarcations that were clinically-significant $\left(\geq 0.5 \mathrm{~cm}^{3}\right)$ and appeared in consecutive slices. 


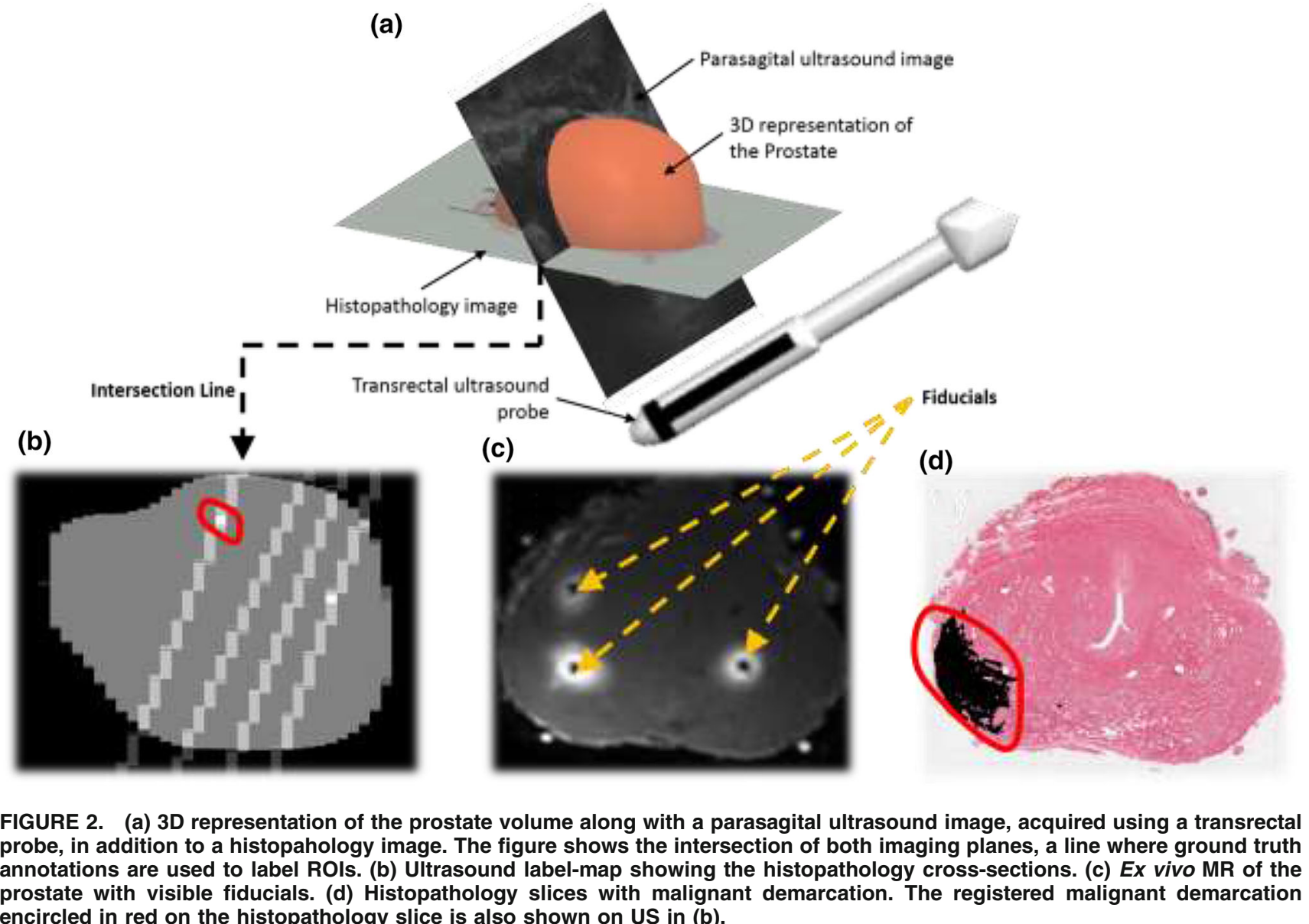
encircled in red on the histopathology slice is also shown on US in (b).

\section{Data Representation}

Each ROI in our dataset is represented as a timeseries, in which each value summarizes the ROI status at a specific time $t$. To produce a single value summary of an ROI at a time-point $t$, we average the $88 \mathrm{RF}$ values within each grid-window in a single frame recorded at the corresponding time $t$. The total number of ROIs selected from the 12 patients is 1100 , where 263 are malignant and 837 are benign. Table 1 summarizes the distribution of ROIs among the 12 patients. Notably, for patients 9-12, only benign ROIs were selected for analysis due to lack of clinically-significant malignant regions. For each ROI, $R_{x}$, a 128long time series, $R_{x}^{y}=R_{x_{1}}^{y}, \ldots, R_{x_{128}}^{y}$, is created, where $y$ indicates the malignancy label assigned to the ROI ( $y$ $\in\{m, b\} ; m$ for malignant, $b$ for benign), and $x$ indicates the ordinal number of the ROI, in the range $1 \leq x \leq 263$ for malignant ROIs, and $1 \leq x \leq 837$ for benign ROIs. Each point $R_{x_{t}}$ in the series corresponds to the average intensity of that ROI in the RF-frame recorded at time $t$, where $1 \leq t \leq T$, and $T=128$. We note that while the number of patients is relatively low, the total number of ROIs per patient is significantly high (see Table 1), thus providing sufficient data to support effective model-learning.

Since we are interested in capturing the patterns of echointensity changes over time, we map the series associated with each ROI, $R_{x}^{y}$, to its respective firstorder difference series, i.e. the sequence of value-differences between pairs of consecutive time-points. Training HMMs that are based on discrete observation symbols rather than on continuous values typically leads to more accurate models with fewer parameters and-more importantly - easily interpretable models. ${ }^{29}$ The latter is particularly advantageous in computer-assisted diagnosis. Hence, we opt for discrete HMMs and represent each TeUS signal as a sequence of distinct observation-values. Since the difference series consist of real-valued numbers, we further quantize the series by placing the values into $M$ equally-spaced bins, where the values in the lowest bin are all mapped to 1 , while those at the top-most bin are mapped to $M$. The resulting set of observations thus consists of bin numbers $(1, \ldots, M)$. In our experiments, we varied the number of bins, $M$, from 10 to 50 , 
TABLE 1. The distribution of malignant and benign ROls over patients.

\begin{tabular}{|c|c|c|c|c|c|c|c|c|c|c|c|c|c|c|}
\hline Patient & Number & 1 & 2 & 3 & 4 & 5 & 6 & 7 & 8 & 9 & 10 & 11 & 12 & Total \\
\hline \multirow[t]{2}{*}{ Number of ROls } & Malignant & 42 & 17 & 64 & 29 & 35 & 18 & 28 & 30 & 0 & 0 & 0 & 0 & 263 \\
\hline & Benign & 42 & 17 & 61 & 29 & 35 & 18 & 29 & 30 & 71 & 254 & 62 & 189 & 837 \\
\hline
\end{tabular}

where the smaller the number of bins - the more lossy the representation is.

The sequence obtained by quantization is denoted $R_{x}^{y^{\prime}}=O_{x_{1}}, \ldots, O_{x_{T-1}}$, where each $O_{x_{t}}$ is the quantized difference in echointensity $\left(R_{x_{t+1}}^{y}-R_{x_{t}}^{y}\right), 1 \leq O_{x_{t}} \leq M$, and $1 \leq t \leq T-1$. In our experiments, we compare the classification performance of HMMs while varying the number of bins used for quantization. The quantized signals are utilized as training and test data for building the models, which are used to distinguish between tissue types as described in the next subsection.

\section{Probabilistic Modeling}

HMMs are often used to model temporal sequences where the generating process is unknown and exhibits variation and noise. ${ }^{11}$ A simplifying assumption underlying the use of HMMs is the Markov property, namely, that the state of the generating process at a given time-point depends only on the state at the preceding point, conditionally independent of all other time points. The states underlie the estimated hidden stochastic-process that emits the observed values in the modeled sequences. An adequate number of states is important to capture both recurrence and variation in patterns along the time series. A model thus requires a sufficient number of states to relay the information conveyed by the sequence. The states, in turn, are associated with distinct emission probability distributions.

An observation corresponds here to the difference in tissue response values recorded in between two consecutive RF-frames and quantized as discussed above. The set of binned echointensity-difference values are the observation symbols that make up the model's alphabet. We assume that the generating process of these echointensity-difference values is unobservable and we estimate it using the observations in the firstorder-difference TeUS signals. The size of the alphabet is the number of available observation symbols, here bins. The number of symbols reflects the level of detail the data representation preserves. Here, we hypothesize that the patterns of change in echointensity over time are the source of tissue-specific information represented by TeUS.

Formally, an HMM $\lambda$ consists of a set of $N$ states, $S=\left\{s_{1}, \ldots, s_{N}\right\}$, an alphabet, $V=\left\{v_{1}, \ldots, v_{M}\right\}$, of $M$ observations, an $N \times N$ stochastic matrix, $A$, governing the state transition probabilities, an $N \times M$ stochastic-emission matrix, $B$, denoting the probability of observing $v_{m}$ at $s_{i}$, and an $N$-dimensional stochastic vector, $\Pi$, that determines the probability to start the process at state $\mathrm{s}_{\mathrm{i}}$. Given a sequence of observations, $O=o_{1}, o_{2}, \ldots, o_{128}$, a model $\lambda$ is learned from the sequence by optimizing the parameters, $A, B$, and $\Pi$, to maximize $\log [\operatorname{Pr}(O \mid \lambda)]$, the probability of the observations $O$ given the model $\lambda$-whose set of parameters are denoted as $\theta$. Given a training set of signals $\left(R^{\prime}\right)$, the learning process aims to find the set of parameters $\theta^{*}$ that maximizes the likelihood of the training set, such that:

$$
\operatorname{Pr}\left(R^{\prime} \mid \theta^{*}\right)=\arg \max _{\theta}\left(\prod_{1 \leq x \leq \mathrm{X}} \operatorname{Pr}\left(R_{x}^{\prime} \mid \theta\right)\right),
$$

where $1 \leq x \leq X$ and $X$ is the total number of ROIs in the training set.

The optimization is done using the Expectation Maximization (EM) method known as the BaumWelch algorithm. ${ }^{24}$ In our experiments, we fix $\Pi$ such that: $\quad \pi_{1}=\operatorname{Pr}\left(\right.$ state $\left._{1}=s_{1}\right)=1$, and $\pi_{j}=0, \forall j \neq 1$. Hence, $s_{1}$ is always the first state.

To learn a model, its parameters are initialized, and then iteratively updated until convergence, in accordance with the EM algorithm. We initialize the model parameters based on clustering the values within all of the training sequences being modeled into $N$ clusters $c_{1}, \ldots, c_{N}$, where $\mathrm{N}$ corresponds to the number of states in the model. Here, clustering is done using $K$ means, with $K=$ the number of states.

\section{Tissue Characterization Framework}

To characterize tissue samples as either benign or malignant, we learn two HMMs- $\lambda_{M}$, for series of malignant tissues, and $\lambda_{B}$ for series of benign tissues. An alternative possible approach is to model both types of tissues through a single HMM, while setting a likelihood threshold to determine the tissue-class. The latter approach requires much empirical analysis to determine both the model parameters and the proper threshold. We thus adopt a two-HMM approach to adequately capture the difference in echointensity pattern. We use supervised learning to optimize the 
model parameters, where the training and test data consist of the TeUS signals corresponding to the ROIs that were labeled as malignant and benign (described in "Data Representation"). Using the training set of malignant ROIs, we learn $\lambda_{M}$, while $\lambda_{B}$ is built using the training set of benign ROIs. For every test-sequence, $\mathrm{ROI}_{\text {test }}$, a log probability is assigned by each of the models $\lambda_{\mathrm{B}}$ and $\lambda_{B}$, and defined as $\log \left(\operatorname{Pr}\left(\mathrm{ROI}_{\text {test }} \mid \lambda_{\mathrm{c}}\right)\right)$, and $c \in\{M, B\}$. This measure indicates how likely the model is to have generated the time-series. The differentiation between benign and malignant tissue is based on the pattern of change between various echointensity ranges and it is determined by the log-probability calculated by the HMMs using the transition and emissions probabilities. Class label $C_{\text {test }}$, assigned to $\mathrm{ROI}_{\text {test }}$, is assigned by the model that generates the maximum log probability, that is:

$$
C_{\text {test }_{\mathrm{i}}}=\underset{c \in\{M, B\}}{\operatorname{argmax}}\left(\log \left(\operatorname{Pr}\left(R O I_{\text {test }_{i}} \mid \lambda_{\mathrm{c}}\right)\right)\right),
$$

where $1 \leq i \leq L$, and $L$ is the number of test-ROIs.

The HMMs used here are all ergodic, consisting of $N$ states and $M$ observations. We assessed the performance of the models while varying the number of states (7 values: $2-8$ states) and the alphabet sizes (5values:10, 20, .., 50).

\section{Cross-validation and Performance Evaluation}

To train each model, we use a leave-one-patient-out cross-validation framework, to account for the nonindependence of ROIs selected from the same patient. We leave-out all the ROIs selected from one patient to be used for testing, and we use the ROIs of the remaining patients for training. We partition each ROI set of TeUS signals (malignant for $\lambda_{M}$, benign for $\lambda_{B}$ ) into training and test sets. In each cross-validation run, the ROIs of one of the 12 patients are left-out as a testset, while the ROIs of the other 11 patients are used to train the HMM. In each cross-validation iteration a pair of models, a malignant $\lambda_{M}$ and a benign $\lambda_{B}$, is trained on the data obtained from the 11 patients and tested on the dataset associated with the left-out patient.

To assess our method's performance, we apply each of the trained models (each trained over ROI timeseries obtained from 11 of the patients) to assign labels to the test data (the ROIs of the left-out 12th patient). We then calculate the average accuracy, sensitivity and specificity of the assigned labels with respect to the ground-truth, where:

$$
\left\{\begin{array}{l}
\text { accuracy }=\frac{\# \text { of correctly classified ROIs }}{\# \text { total }- \text { ROIs }} ; \\
\text { sensitivity }=\frac{\# \text { correctly classified malignant ROIs }}{\# \text { of malignant ROIs }}
\end{array} ; .\right.
$$

To report diagnostic performance, we also plot the receiver operating characteristic (ROC) curves and calculate the Area Under the Curve (AUC) for each of the 8 patients, who contributed malignant and benign ROIs to the dataset (Fig. 5). The ROC curves are generated using the $\log$ odds ratio (denoted $\log (\mathrm{OR})$ ) that is the log of the ratio of the likelihood value from models of benign signals and that from models of malignant signals. $\log (\mathrm{OR})$ greater than a classification-threshold of one indicates a prediction of a benign label which is equivalent to $C_{\text {test }_{i}}=B$ in Eq. (2). To generate an ROC curve for a left-out patient, we first normalize the $\log (\mathrm{OR})$, calculated for every test ROI, to have values $\in[0,1]$ and a classification-threshold of 0.5 . We then plot the true positive rates (sensitivity) vs. false positive rates (1-specificity) calculated for various classification-thresholds with values ranging between zero and one.

To employ our system in practice, we provide the physician performing the biopsy with ultrasound images overlaid with colormaps, where the latter highlight areas that are more likely to be cancer, and should be targeted during biopsy. The color of each ROI in the colormap (see Fig. 6) is determined by the $\log$ odds ratio. The $\log (\mathrm{OR})$ reflects the confidence of the label prediction. When the difference between the probabilities, generated by both HMMs, is very small, the $\log (\mathrm{OR})$ is approximately zero reflecting a very low confidence in labeling. The more different the probabilities are the higher is the confidence in the assigned label and the further away from zero is the $\log (\mathrm{OR})$. The range of $\log (\mathrm{OR})$ is calculated for the TeUS signals in each test set and mapped to a spectrum of colors ranging from blue for negative values, green/ yellow for values close to zero, and orange/red for the positive values.

\section{RESULTS}

As noted above, we experimented with seven different values for the number of states, $N,(2 \leq N \leq 8)$, and five values for alphabet size, $M$ (from 10 to 50). For each combination of $N$ and $M$, the structure of the HMMs are learned through 12 cross-validation itera- 
tions, where in each iteration the ROIs of one of the 12 patients are left-out for testing, while the ROIs of all other patients are used for training. Twelve pairs of models are therefore trained for each combination of $N$ and $M$, one pair per cross-validation iteration. We compared the outcome of the $35(7 \times 5)$ experiments to select the HMMs showing best performance while prioritizing parsimonious models, (i.e. those that use fewer parameters, while retaining the same level of performance).

Table 2 shows the resulting percent-accuracy values for the 35 experiments. The topmost average accuracy values, shown in boldface in Table 2, were attained by HMMs comprising 4 states and 40 observations $(85.35 \%), 6$ states and 10 observations $(85.15 \%)$, and 5 states and 50 observations $(85.06 \%)$. These three values are not statistically significantly different from one another. However, the models comprising 6 states and 10 observations are the most parsimonious, thus we select them to generate the colormaps and ROC curves shown in Figs. 5 and 6.

A graphical representation of the pair of HMMs that have 6 states and 10 observations (the most parsimonious model with classification accuracy of $85.15 \%$ ) is shown in Fig. 3, to help in visualizing the classification process. Figure 3 shows the pair of 6state HMMs, where the left one was trained on timeseries obtained from malignant ROIs while the one on the right was trained on benign signals. The transition probabilities are shown on the edges while emission probabilities for each state are shown as histograms. The figure shows that in both models, each state is characterized by its own markedly distinct observation distribution. The clear distinction between the two models means that TeUS signals of malignant ROIs has patterns of changes different than those of benign ROIs.

Table 3 lists the accuracy, sensitivity, specificity and AUC attained by the most parsimonious models (6states and 10-observations) for the patients in our dataset. Notably, as no clinically malignant regions were originally identified in patients P9-P12 (see "Data Representation" and Table 1), no corresponding malignant ROIs are available and as such the sensitivity and AUC values are not shown for these patients. Compared to previously published results reporting an average accuracy of $80 \%$ on the same dataset, ${ }^{12}$ the values shown in Table 2 all indicate improved performance. Notably, not all the differences between previous results and those reported in the tables are statistically significant, due to variability in performance across different patients. Statistically significant increase in accuracy with respect to previously published results $(p$-values $<0.05$, one tailed Mann-Whitney-Wilcoxon test), was attained by the 3- state HMMs with 30 and 40 observations, the 4-state HMMs with 40 observations, and the 7-state models with 10 observations. The performance of the model with 5 states and 50 observations was further improved by noise injection, where accuracy reached $85.6 \%$ with an AUC of 0.95 (this improvement is also statistically significant, $p$-value $<0.04)$.

To show that the models captured the difference between the patterns of echointensity changes of benign and malignant tissues, we compare the emission probabilities of the malignant HMM with those of the benign model. For this comparison, we plot the maximum emission probabilities of both HMMs for each observation symbol, regardless of the emitting state. In Fig. 4, the solid red bars show the maximum emission probability per observation for the malignant model and the diagonally striped blue bars for the benign model. The plot clearly shows that the benign model assigns higher probabilities than the malignant HMM to the first 4 observations as well as to the eighth one (negative echointensity differences $\leq-3$; positive values between 5 and 7 , see the tissue characterization framework for details).

To demonstrate the trade-off between sensitivity and specificity for the most parsimonious HMM (6 states and 10 observations), we plotted ROC curves and calculated AUC values. Figure 5 shows the ROC curves for 8 patients from whom malignant and benign ROIs were taken. All of the curves lie well above the diagonal line, indicating a balanced trade-off between true positives and true negatives. The curve closest to the diagonal shows the performance of testing the ROIs of patient P8, who has the lowest sensitivity. The poorer performance for this patient is likely due to higher registration errors between the ultrasound data and histopathology labels. It should be noted that all of the patients in our data went through prostatectomy as part of their clinical-care plan. It is possible that some of the false positives, are not entirely erroneous as TeUS might have relayed information about malignancy outside of the histophathology cross-sections.

To visualize the labeling results on the ultrasound images, we generated colormaps for each RF-frame by color-coding the ratio of log probabilities calculated by the models. Figure 6 shows examples of RF-frames obtained from the 12 patients. Two versions of the same RF-frame are displayed; images on the left show histopathology cross-sections and labeled ROIs selected for analysis, whereas the images on the right show the corresponding colormaps based on the probability values assigned by the HMMs. Each ROI is assigned a color reflecting the log-odds ratio calculated for its respective time series (see Cross-validation and Performance Evaluation). Only a few ROIs have 
TABLE 2. Tissue-classification accuracy along with standard deviation (in parentheses) for HMMs as a function of varying state number and alphabet size.

\begin{tabular}{|c|c|c|c|c|c|c|c|}
\hline \multirow[b]{2}{*}{ Alphabet size } & \multicolumn{7}{|c|}{ Number of states } \\
\hline & 2 & 3 & 4 & 5 & 6 & 7 & 8 \\
\hline 10 & $82.28(10.7)$ & $82.62(13.2)$ & $82.81(11.04)$ & $83.67(10.5)$ & $85.15(10.5)$ & $84.67^{*}(10.4)$ & $83.11(8.8)$ \\
\hline 20 & $83.23(11.3)$ & $83.63(10.9)$ & $83.52(9.9)$ & $83.26(10.5)$ & $83.08(10)$ & $84.92(11.1)$ & $84.12(9.7)$ \\
\hline 30 & $84.45(10.8)$ & $84.9^{*}(11)$ & $83.07(11.4)$ & $82.73(11.2)$ & $84.57(10.1)$ & $84.32(10.8)$ & $84.8(10.9)$ \\
\hline 40 & $84.64(10.1)$ & $84.63(10.7)$ & $85.35^{\star}(9.7)$ & $84.35(11.2)$ & $83.74(9.8)$ & $84.45(10.8)$ & $84.78(9.3)$ \\
\hline 50 & 83.54 (11.9) & $83.72(10.1)$ & 82.72 (10.3) & $85.06(10.7)$ & $83.34(10.4)$ & $83.26(8.8)$ & $84(9.9)$ \\
\hline
\end{tabular}

The three highest accuracy values are shown in boldface. Asterisks indicate values that are statistically significantly higher than the state-ofthe-art on the same dataset.

(a) Malignant HMM

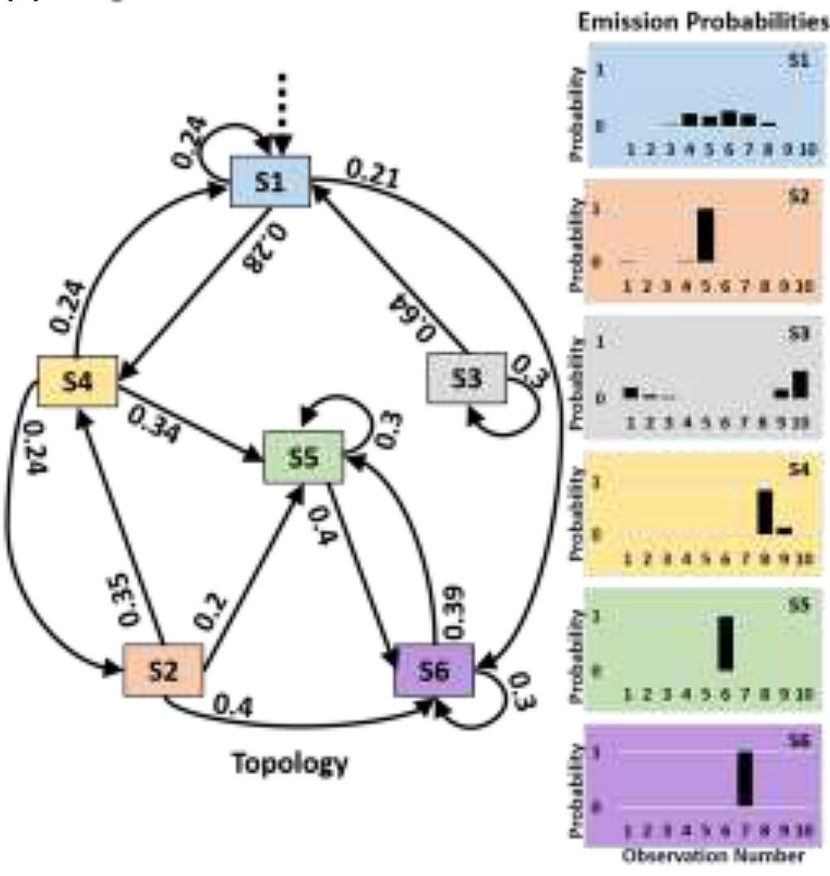

(b) Benign HMM

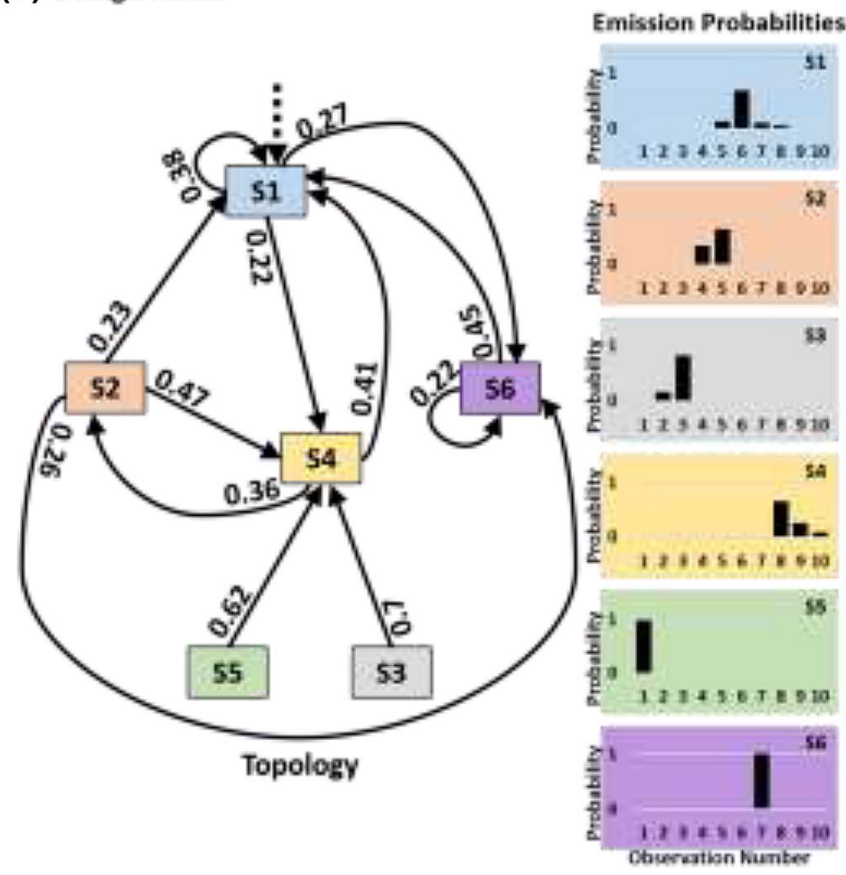

FIGURE 3. A graphical representation of the HMMs that have 6 states, 10 observations, and an accuracy of $85.15 \%$. (a) The HMM learned from malignant ROIs, and (b) the HMM trained on benign ROIs. Nodes represent states. Edges are labeled by transition probabilities; Emission probabilities are shown to the right of each model. Edges with probability $<0.2$ are not shown. This figure demonstrates the distinct emission and transition probabilities learned by each of the models.

ground truth labels shown in the corresponding images on the left-hand side. The sparsity of true labels is due to the slicing protocol adopted for whole-mount histopathology analysis. Our method assigned labels to all the ROIs within the boundaries of the prostate (with or without ground-truth) and assigned them colors accordingly. The color-maps are therefore used to inspect the overall patterns of prediction for ROIs without true labels. They help in comparing our predictions to the known statistics about the frequency of malignancy occurring in each of the prostate zones.

The colormaps of RF-frames from patients P1-P7 match the true-annotations almost perfectly. The col- ormap associated with patient P8 shows more false negatives, which agrees with the performance measures shown in Table 3. As for patients P9-P12, the colormaps show few false positives compared to the true labels (shown to the left of each colormap) showing no malignant ROIs. It should be noted though that patients P9-P12 went through prostatectomy due to cancerous samples in their biopsies. Hence, it is possible that some of what appear to be false positives are actually true positives, and the TeUS has indeed relayed correct information about malignancy outside the histopathology cross-sections used for denoting the ground truth. 
TABLE 3. Classification performance using 6-state HMMs with an alphabet size of 10 .

\begin{tabular}{lcccc}
\hline & Accuracy & Sensitivity & Specificity & AUC \\
\hline P1 & 92.9 & 100 & 85.7 & 0.99 \\
P2 & 82.4 & 70.6 & 94.1 & 0.96 \\
P3 & 89.6 & 79.7 & 100 & 0.98 \\
P4 & 96.6 & 93.1 & 100 & 0.99 \\
P5 & 91.4 & 100 & 82.9 & 0.99 \\
P6 & 94.4 & 88.9 & 100 & 1 \\
P7 & 84.2 & 78.6 & 89.7 & 0.92 \\
P8 & 71.7 & 53.3 & 90 & 0.83 \\
P9 & 97.2 & - & 97.2 & - \\
P10 & 85.4 & - & 85.4 & - \\
P11 & 74.2 & - & 74.2 & - \\
P12 & 61.9 & - & 61.9 & - \\
Average & 85.15 & 83 & 88.4 & 0.95 \\
\hline
\end{tabular}

The symbol "-" denotes missing sensitivity and AUC values. These values cannot be calculated for patients P9-P12 since all ROIs for those patients were benign (see Table 1). The bottom row provides the average along each measure, calculated over the values shown; Sensitivity and AUC are averaged only over patients P1-P8, while all other measures are averaged over P1-P12.

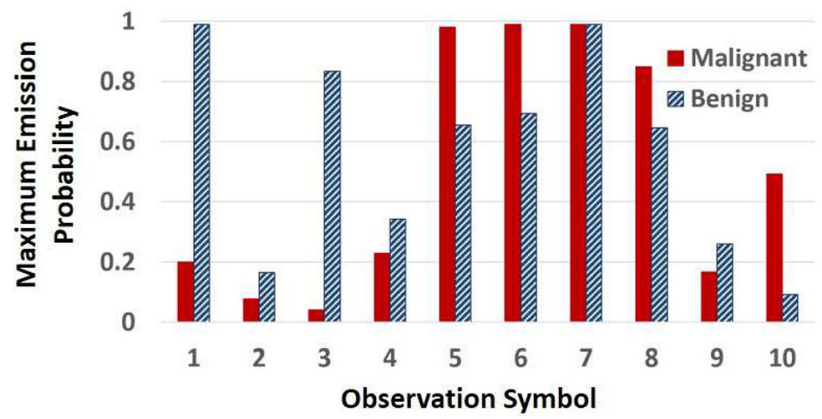

FIGURE 4. Maximum emission probabilities per observation (irrespective of the emitting state) for each of the malignant (solid red) and benign (striped blue) HMMs. This figure shows which model, malignant or benign, has a higher emission probability for each symbol in the alphabet regardless of the emitting states.

\section{DISCUSSION}

Image-guided detection of prostate cancer draws a significant amount of research interest. In this paper, we introduced stochastic models to improve the detection and stratification of PCa using TeUS, an innovative ultrasound-based imaging technology. We explicitly captured the temporal aspect of tissue-responses to prolonged sonication through HMMs. Our results show that ROIs of malignant tissues go through a different state sequence in comparison with the ROIs of benign tissue. These findings are in concordance with Bayat et al.'s study reporting that TeUS captures the micro-structure of tissues. They identified the dominant phenomenon governing the interactions between TeUS and the scanned tissue as micro-vibrations of $1-2 \mathrm{~Hz}$ frequency (related to pulsation)3. Hence

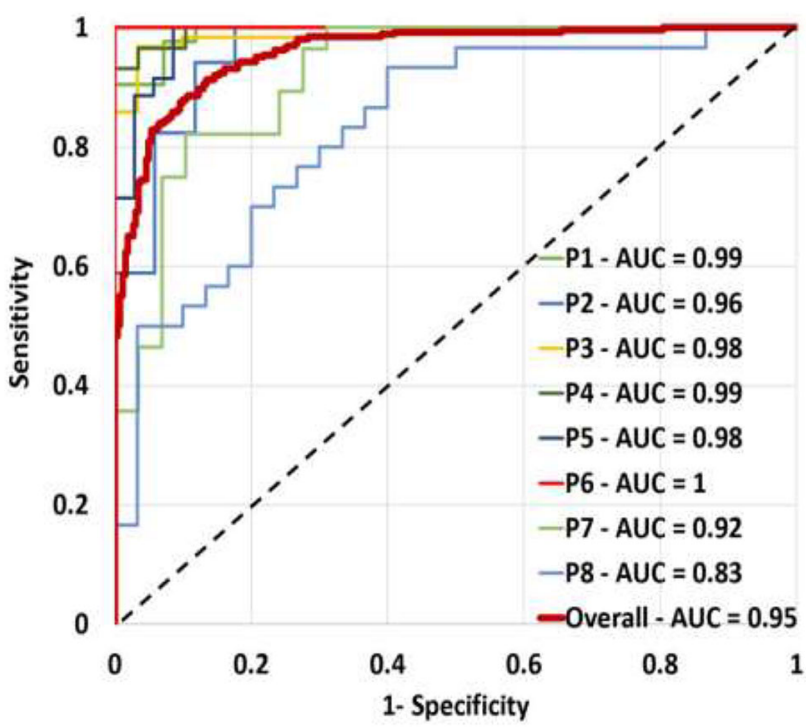

FIGURE 5. ROC Curves showing the performance perpatient of the respective HMM-trained while leaving out the ith patient data and used to predict the ROI labels of that leftout patient. The AUC value for each of the ROC curves is shown in the legend. The bold red curve indicates the overall performance, summarizing the trade-off between sensitivity and specificity while assigning the ROI labels for all eight patients.

incorporating the temporal nature of the signals in TeUS models enables the HMMs to capture the periodicity of micro-vibrations affecting the microstructure of tissues, and in turn, causing changes in echointensity.

Our models lead to a statistically significant improvement in prostatic-tissue classification, showing that the time-domain of TeUS carries informative data. As demonstrated by accurate differentiation between malignant and benign TeUS signals, the models capture tissue-specific patterns of echointensity changes. Thus our HMMs effectively represent the different temporal signatures of malignant vs. benign tissue.

To facilitate a seamless clinical translation with minimal interruption to current diagnostic procedures, we investigated the effect of model parameterization on the classification outcome. We determined the most parsimonious models through comparing the performance of HMMs varying in number of states and alphabet size. The adequate number of states and alphabet is specific to the data being modeled, since the structure of an HMM is related to the patterns and motifs available in the training sequences. To generalize these findings beyond our data, the HMMs need to be trained on TeUS signals from more patients. Parsimonious models can be efficiently implemented for real-time applications; thus, using them can support clinical deployment of TeUS. Our results indicate 


\section{Benign}

Malignant
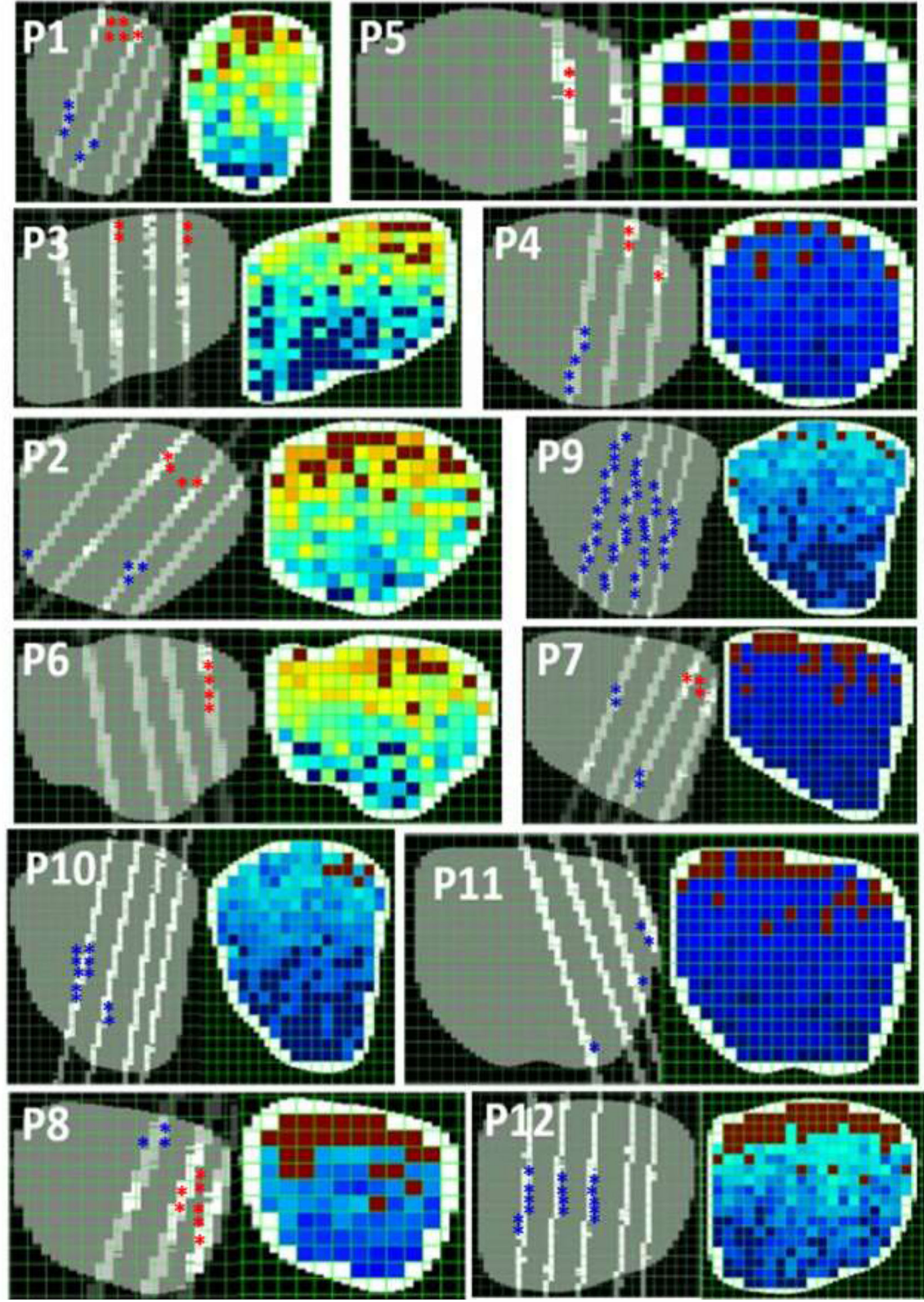

BMES 
4FIGURE 6. Colormaps of the RF-frames overlaid with malignant/benign pathology labels obtained for each of the patients. For each patient, the left image shows the histopathology cross-section (shown in oblique lines with white regions denoting histopathology demarcations) along with the selected ROls per patient (red asterisks for malignant ROls and blue ones for benign), while the right image shows the color-maps based on the HMM probability assignments. Shades of red indicate higher malignancy while blue indicates lower malignancy.

that the captured temporal patterns help differentiate between malignant and benign TeUS time series, where the best achieved accuracy was $85.6 \%$ along with an AUC of 0.95 . These results improve over previously published results $(80 \%$ accuracy and an AUC of 0.93$)$, where our accuracy $(85.6 \%)$ is statistically significantly larger than the one reported by Imani et al. ( $p$-value $<$ 0.04). ${ }^{12}$ Despite this improvement, we had false positives reflected in Table 3 and Fig. 6. Possible reasons for the poorer performance are ROI mislabeling, higher registration errors, or over-fitting the training, where test data exhibits different patterns of changes than the training set.

The colormaps, shown in Fig. 6, reveal a color pattern, where colors encoding malignancy is often present at the top of the RF-frames and colors representing benign regions appear at the bottom. This pattern may be attributed to multiple factors. The top of the images are in the peripheral zone of the prostate, closer to the rectum, where there is much higher chance of having cancerous lesions. More than $75 \%$ of prostate cancer arise in the peripheral zone whereas $\sim 20 \%$ appear in the transitional zone and only $5-8 \%$ in the central zone. ${ }^{23,25}$ It is important to note that after data collection, we became aware that inconsistent depthdependent time-gain compensation was applied during imaging. This inconsistency might have also affected the color patterns shown in Fig. 6. For future work, we are planning to adopt a transfer learning technique, where we incorporate in our models knowledge acquired by other models trained on signals collected while using appropriate time-gain compensation.

Our findings show that TeUS is a promising imaging technique. The generated cancer likelihood maps can be used for patient-specific targeting during prostate biopsies and increase the yield of this procedure for detecting clinically significant prostate cancer. Since TeUS is based on conventional ultrasound, it is cost effective, widely available and accessible, and requires a minimal amount of training for practitioners.

Our study is a first step toward using HMMs to model TeUS signals, where a large number of ROIs (1100) is used for adequate modeling using HMMs despite the relatively small number of patients. As additional high-quality data becomes available, we plan to increase the number of patients, and include anatomical-data indicating the zones from which ROIs are selected. We expect our method to be applicable to other types of cancer where ultrasound is part of conventional diagnostic workflow such as breast and liver to assist in more accurate and timely detection of disease.

\section{ACKNOWLEDGMENTS}

This work was partially supported by Grants from NSERC Discovery to Hagit Shatkay and Parvin Mousavi, NSERC and CIHR CHRP to Parvin Mousavi and NIH R01 LM012527, U54 GM104941 and NSF IIS EAGER Grant \#1650851 to Hagit Shatkay.

\section{CONFLICT OF INTEREST}

The authors declare that they have no conflict of interest.

\section{OPEN ACCESS}

This article is licensed under a Creative Commons Attribution 4.0 International License, which permits use, sharing, adaptation, distribution and reproduction in any medium or format, as long as you give appropriate credit to the original author(s) and the source, provide a link to the Creative Commons licence, and indicate if changes were made. The images or other third party material in this article are included in the article's Creative Commons licence, unless indicated otherwise in a credit line to the material. If material is not included in the article's Creative Commons licence and your intended use is not permitted by statutory regulation or exceeds the permitted use, you will need to obtain permission directly from the copyright holder. To view a copy of this licence, visit http://crea tivecommons.org/licenses/by/4.0/.

\section{REFERENCES}

${ }^{1}$ American Cancer Society. Cancer Facts \& Figures 2019. Atlanta: American Cancer Society, 2019.

${ }^{2}$ Azizi, S., F. Imani, et al. Detection of prostate cancer using temporal sequences of ultrasound data: a large clinical feasibility study. Int. J. Comput. Assist. Radiol. Surg. 11:947-956, 2016.

${ }^{3}$ Bayat, S., S. Azizi, et al. Investigation of physical phenomena underlying temporal-enhanced ultrasound as a new diagnostic imaging technique: theory and simulations. 
IEEE Trans. Ultrason. Ferroelectr. Freq. Control 65:400 410, 2018.

${ }^{4}$ Boyd, L. K., X. Mao, and Y. J. Lu. The complexity of prostate cancer: genomic alterations and heterogeneity. Nat. Rev. Urol. 9:652-664, 2012.

${ }^{5}$ Carter, H. B., P. C. Albertsen, et al. Early detection of prostate cancer: AUA guideline. J. Urol. 190:419-426, 2013.

${ }^{6}$ Coast, D. A., R. M. Stern, et al. An approach to cardiac arrhythmia analysis using hidden markov models. IEEE Trans. Biomed. Eng. 37:826-836, 1990.

${ }^{7}$ Cook, E. D., and A. C. Nelson. Prostate cancer screening. Curr. Oncol. Rep. 13:57-62, 2011.

${ }^{8}$ Etzioni, R., A. Tsodikov, et al. Quantifying the role of PSA screening in the US prostate cancer mortality decline. Cancer Causes Control 19:175-181, 2008.

${ }^{9}$ Gibson, E., C. Crukley, et al. Registration of prostate histology images to ex vivo MR images via strand-shaped fiducials. J. Magn. Reson. Imaging 36:1402-1412, 2012.

${ }^{10}$ Graif, T., S. Loeb, et al. Under diagnosis and over diagnosis of prostate cancer. J. Urol. 178:88-92, 2007.

${ }^{11}$ Hauskrecht, M., and H. Fraser. Planning treatment of ischemic heart disease with partially observable Markov decision processes. Artif. Intell. Med. 18:221-244, 2000.

${ }^{12}$ Imani, F., P. Abolmaesumi, et al. Computer-aided prostate cancer detection using ultrasound RF time series: in vivo feasibility study. IEEE Trans. Med Imaging 34:2248-2257, 2015.

${ }^{13}$ Inal, G., V. Oztekin, et al. Sixteen gauge needles improve specimen quality but not cancer detection rate in transrectal ultrasound-guided 10-core prostate biopsies. Prostate Cancer Prostatic Dis 11(3):270-273, 2008.

${ }^{14}$ Khalil, A. S., R. C. Chan, et al. Tissue elasticity estimation with optical coherence elastography: toward mechanical characterization of in vivo soft tissue. Ann. Biomed. Eng. 33:1631-1639, 2005.

${ }^{15} \mathrm{Li}$, Y., S. LipskyGorman, et al. Section classification in clinical notes using supervised hidden markov model. Proc of ACM Int Health Informatics Symp, pp. 744-750, 2010.

${ }^{16}$ Loeb, S., M. Bjurlin, et al. Overdiagnosis and overtreatment of prostate cancer. Eur. Urol. 65:1046-1055, 2014.

${ }^{17}$ Moradi, M., P. Abolmaesumi, et al. Augmenting detection of prostate cancer in transrectal ultrasound images using SVM and RF time series. IEEE Trans. Biomed. Eng. 56:2214-2224, 2009.

${ }^{18}$ Nahlawi, L., C. Goncalves, et al. Stochastic modeling of temporal enhanced ultrasound: impact of temporal properties on prostate cancer characterization. IEEE Trans. Biomed. Eng. 65:1798-1809, 2018.
${ }^{19}$ Nahlawi, L., F. Imani, et al. Using hidden markov models to capture temporal aspects of ultrasound data in prostate cancer. Proc IEEE Int Conf Biomed Informatics and Biomedicine, pp. 446-449, 2015.

${ }^{20}$ Nahlawi, L., F. Imani, et al. Prostate cancer: improved tissue characterization by temporal modeling of radio-frequency ultrasound echo data. Proc Int Conf Med Image Comput and Computer-Assisted Interv, pp. 644-652, 2016.

${ }^{21}$ Öbek, C., T. Doğanca, et al. Core length in prostate biopsy: size matters. J. Urol. 187:2051-2055, 2012.

${ }^{22}$ Pinto, P. A., P. H. Chung, et al. Magnetic resonance imaging/ultrasound fusion guided prostate biopsy improves cancer detection following transrectal ultrasound biopsy and correlates with multiparametric magnetic resonance imaging. J. Urol. 186:1281-1285, 2011.

${ }^{23}$ Pullar, B., and N. Shah. Prostate cancer. Surgery (Oxford) 34(10):505-511, 2016.

${ }^{24}$ Rabiner, L. R. A tutorial on hidden markov models and selected applications in speech recognition. Proc IEEE 77:257-286, 1989.

${ }^{25}$ Scher, H.I., S. Leibel, et al. Cancer of the prostate. In: DeVita, Hellman, and Rosenberg's Cancer: Principles and Practice of Oncology, 10th ed. Lippincott Williams \& Wilkins, Philadephia, pp. 932-980, 2015.

${ }^{26}$ Singer, E. A., A. Kaushal, et al. Active surveillance for prostate cancer: past, present and future. Curr. Opin. Oncol. 24:243-250, 2012.

${ }^{27}$ Sonn, G. A., E. Chang, et al. Value of targeted prostate biopsy using magnetic resonance-ultrasound fusion in men with prior negative biopsy and elevated prostate-specific antigen. Eur. Urol. 65:809-815, 2014.

${ }^{28}$ Tempany, C., S. Straus, et al. MR-guided prostate interventions. J. Magn. Res. Imaging 27:356-367, 2008.

${ }^{29}$ Wallhoff, F., S. Eickeler, G. Rigoll. A comparison of discrete and continuous output modeling techniques for a pseudo-2D hidden markov model face recognition system. Proc Int Conf Image Processing, vol. 2, pp. 685-688, 2001.

${ }^{30}$ Wang, P., C. S. Lim, et al. Phonocardiographic signal analysis method using a modified hidden Markov model. Ann. Biomed. Eng. 35:367-374, 2007.

${ }^{31}$ Ward, A. D., C. Crukley, et al. Prostate: registration of digital histopathologic images to in vivo MR images acquired by using endorectal receive coil. Radiology 263:856-864, 2012.

Publisher's Note Springer Nature remains neutral with regard to jurisdictional claims in published maps and institutional affiliations. 